\title{
Design of Mechanism for Reducing Support Structures in Material Extrusion Additive Manufacturing
}

\author{
NASSIM KHALED ${ }^{1}$, RAMI ALKHATIB ${ }^{2}$, ANAS AL-SHAGHOURI ${ }^{3}$, SAJA KHATIB ${ }^{4}$ \\ ${ }^{1}$ Mechanical Engineering Department, Prince Mohammad Bin Fahd University, nkhaled@pmu.edu.sa, \\ Al Khobar, Saudi Arabia \\ ${ }^{2}$ Mechanical and Mechatronics Engineering Department, Rafik Hariri University, \\ Mechref, Damour, Lebanon \\ ${ }^{3}$ Mechanical and Mechatronics Engineering Department, Rafik Hariri University, \\ Mechref, Damour, Lebanon \\ ${ }^{4}$ Mechanical and Mechatronics Engineering Department, Rafik Hariri University, khatib- \\ Mechref, Damour, Lebanon
}

\begin{abstract}
Material extrusion additive manufacturing is widely used in constructing 3D parts. Traditional 3D printers create support structures to build some portions of a model with overhangs that are not supported from below. The purpose of this paper is to introduce a mechanism that can reduce the use of support structures required during 3D printing. The robotic arm is made up of links that are connected with both prismatic and rotary joints. The end-effector of this manipulator resembles temporal support. It is used while building some portions of a model instead of printing support structures. To serve this goal, a mathematical model for the robotic arm based on Chasles-Mozzi theorem is introduced to avoid the limitations of Denavit-Hartenbrg

convention. Finally, this paper will present the operation flowchart to be implemented in 3D printers' software. In other words, predefined usage of the mechanism will help to estimate the amount of the material needed for printing the desired object. The ultimate objective behind employing a robotic arm in material extrusion additive manufacturing is to have a printer that produces clean prototypes, consume less material and reduce printing time.
\end{abstract}

Key-Words: Material Extrusion; Additive Manufacturing; Screw Motion; Robotics Arm

Received: March 25, 2020. Revised: September 13, 2020. Accepted: September 30, 2020.

Published: October 8, 2020.

\section{Introduction}

3D printing is a vital topic in additive manufacturing that is capturing both researchers and industries due to the following reasons: 1) Ability to build up components with extraordinary geometrical complexity 2) Modulate the material composition and density 3) Save time during the production phase and testing in addition to the flexibility that 3D printers can add. Supports in 3D printers are a major part of the printing process. Absence or improper supports cause collapses within the part in addition to other distortions. Yet, support structures consume the material that will not be used or needed in the end product. Moreover, more time is consumed while printing supports that can take longer based on the complexity of structure. Besides, additional time is then required for removing support material from prints. Different algorithms are developed to enhance the positioning of support structures within the FDM (Fused Deposition Modeling). In this process, the melted filament is extruded along a predetermined path to form a solid surface that provides the foundation for the next layer of material to be constructed upon the current layer [1]. Furthermore, the necessity of supports depends on the degree of overhang. If the overhang is less than $45^{\circ}$, no support is required. However, if the overhang is more than $45^{\circ}$ then support is needed as given in Fig.1. Likewise, if support structures are needed, it is important to make sure that they are reachable during postprocessing [6].

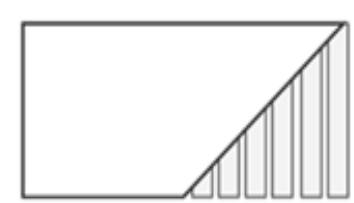

Overhang of more than 45 degrees Support is needed

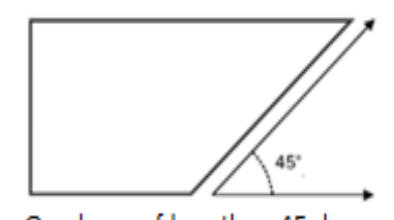

Overhang of less than 45 degrees No support is needed
Figure 1: FDM support structure 
FDM printing method has two support types: flat accordion and tree-like support. The software reinforces choosing one of the options. However, tree-like support is considered to be better [2]. Newer technologies introduced the dissolvable support in which the support structures can be printed with dissolvable material and can be eliminated using specific chemical solution [3] such as isopropyl alcohol and recently just water. However, this way is expensive and timeconsuming. In the context of additive manufacturing, Greer et al. discussed the design rules excavator arm produced in Metal Big Area Additive Manufacturing including the overhanging constraints [4].

Leary et al. focused on modifying the optimal topology of material distribution which cause minor geometric variations to attain support-free additive manufacturing. The authors conducted a case study on manufacturing cantilever beam based on a polymeric additive system. The results achieved an increase in the inclination angle of the modified geometry, reduction in manufacturing time and identification of optimal component orientation. Although the addition of material encountered by the topologically optimal geometry method caused additional load transmission paths, the mass of the print is higher which may not be convenient for some applications [5]. In a further study, mixedlayer adaptive slicing method for robotic additive manufacturing has been implemented. The method combines both non-planar slicing for capturing print details efficiently and planar slicing for less time manufacturing. Such manufacturing intelligence conveyed higher performance in building complex objects [6].

Robotic arms and 3D-printers are in tight relation where parts of the robot are manufactured via 3DPrinters [7] and vice versa. Industrial robotic arms are widely employed in manufacturing non-standard morphologies. Namely, Kontovourkis et al. mounted the extruder of a 3D clay printing control system on industrial robotic arm ABB 600-20/1.65 with IRC5 controller [8]. Ishak et al. developed the concept of using a robotic arm in $3 \mathrm{~d}$ printing for faster prototyping. This is done by giving the nozzle more degrees of freedom and by giving the software capabilities of generating nonplanar toolpath [9]. In another research, the robotic arm has been engaged in building struts on the 3D lattice structure design by performing multi-plane toolpath motions [10]. Mobile printing robots have been developed in another research to print larger structure shapes where the printed object is larger than the printer itself [11].
Accordingly, robot arms are disrupting technologies in additive manufacturing. Namely, cooperative robots can build larger printed sizes with better subtractive process compared to traditional way of 3d-printnig [12]. Therefore, constraints can be reduced on the design size due to chamber volume of 3D printing. The efficiency exceeded $73 \%$ using multi-robot platform (made up of four robots) while printing a model compared to robot-less printing methods [13]. Furthermore, development of robots [14] could improve the printing process. For instance, Carabine et al. optimized the design of springs (based on both dynamic [15] and electro-mechanical model) mounted on a delta robot that yielded an energy efficient technique by reducing $50 \%$ of the energy consumption during 3D printing [16].

Consequently, 3D printing and robotics employed together, interacted and got a tight correlation to attain certain tasks like in flexible electronics [17], wireless communication [17], soft robotics [18], generating robot trajectories for Conformal 3D Printing using nonplanar layer [19], enhancement of the mobile robot position dedicated for habitable house construction by $3 \mathrm{D}$ printing [20], multi-material soft robot that are used on drug delivery applications [21], and concrete printing [22].

In this paper, the integration of the robotic arm to the $3 \mathrm{D}$ printer based on material extrusion additive manufacturing is investigated to reduce the number of printed support structures. The rest of the paper is outlined as follows: a motivation behind the importance of reducing support structures in prints is investigated. Then the recommended mechanism is studied along with the modified 3D printing flowchart. Finally, a conclusion has been constructed on the performance quality of the robotic arm and its functionality in $3 \mathrm{D}$ printing.

\section{Motivation}

FDM is the most common type of 3D printers. The newly proposed mechanism will work on this type of printers. Such a mechanism was made to be flexible and to reach as many places that need support structure as it can be. This comes with the limitation of reducing support structures by changing the printing orientation relative to the build plate.

Two experiments are conducted using the common FDM printer to demonstrate the need for a modification in the printer mechanism. Both experiments are made to print a hollow cube, $8.5 \mathrm{~cm}$ $x 7 \mathrm{~cm} \mathrm{x} 7 \mathrm{~cm}$. We have chosen this model since it can't be built without supports as bridges form the 
main structure. In this case, bridges are connected with an angle of $90^{\circ}$, and there is no previous layer before their construction. In the first experiment, we have printed the cube without the usage of supports as demonstrated in Fig. 2-a.

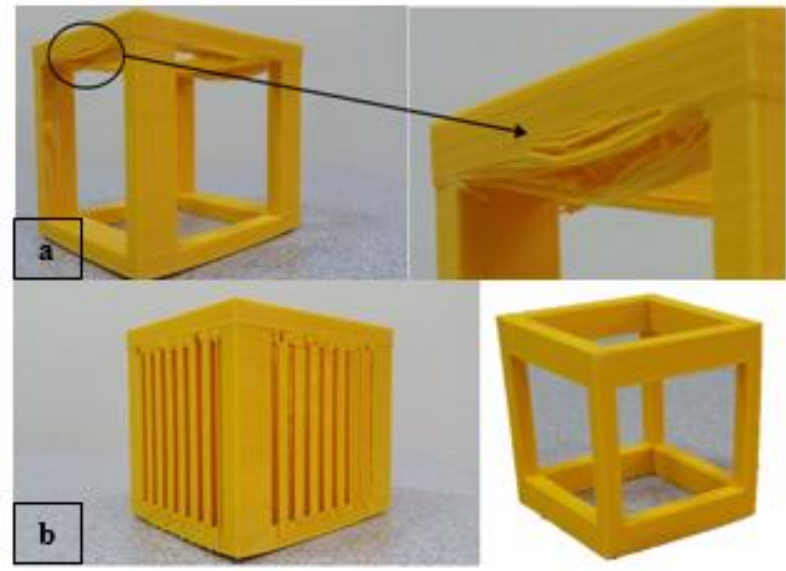

Figure 2: (a) Structural distortion of printed model without support compared to the printed cube with (b) presence of support and after removing the support

Fig. 2 indicates that the construction of the cube is completed even though the printing process is initiated to print the model without supports. However, the quality attained in the finished product was unsatisfactory. The dimensional accuracy and structural quality of the bridge failed to be printed correctly. The variation in the used filament would also influence the outcome more dreadfully. This experiment took 1 hour and 15 minutes.

In the second experiment, the cube is printed with the help of support structures. The structural quality of the cube was as desired and is shown in Fig. 2-b. Thus, the dimensional bridge decent quality was achieved in the presence of support structures. However, this achievement consumed more time and material. It took 2 hours and 45 minutes to print and consumed about twice the amount of material of the first one.

Fig. 3 presents the mass of each cube in the above two experiments.

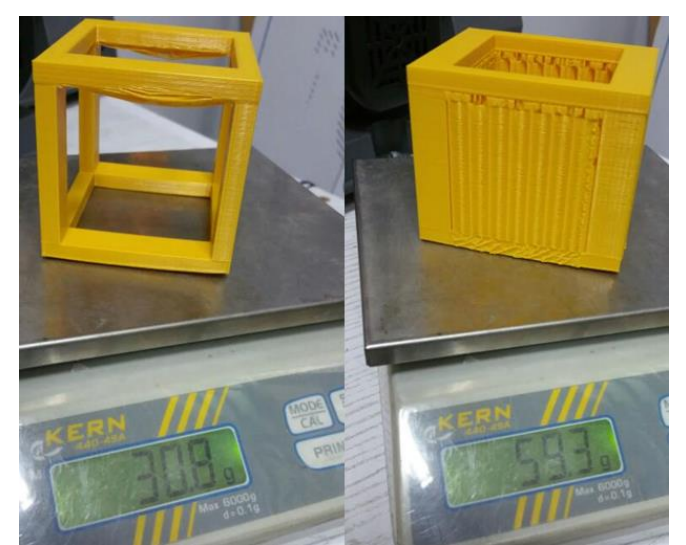

Figure 3: The mass of the printed cube without and with support structures

The mass of supports was approximately equal to the mass of the clean cube. Fig.4 illustrates the cube and the removed support structures. The figure shows clearly that such traditional way of printing using support structures consumes extra material in comparison with the mass of the printed model. Furthermore, such prints would inquire additional time.

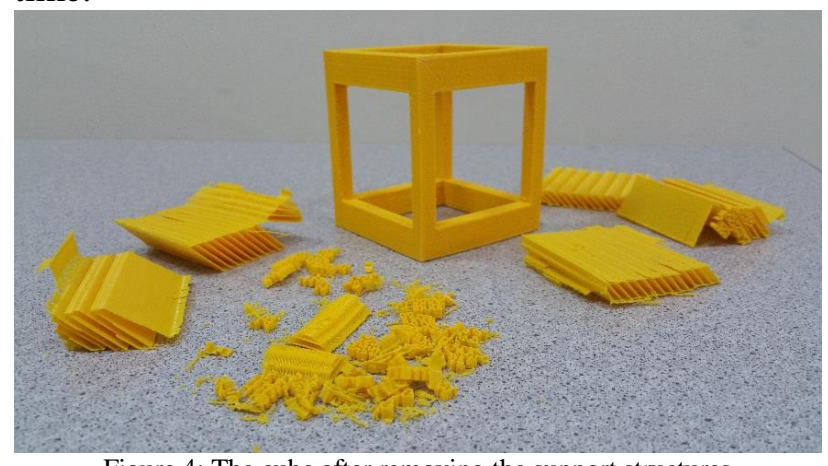

Figure 4: The cube after removing the support structures

\section{Proposed Mechanism}

The newly proposed mechanism that is intended to reduce the problem of support structures must be flexible and can adapt to different types of support that will be needed while building the model. The proposed mechanism is illustrated in Fig. 5. 


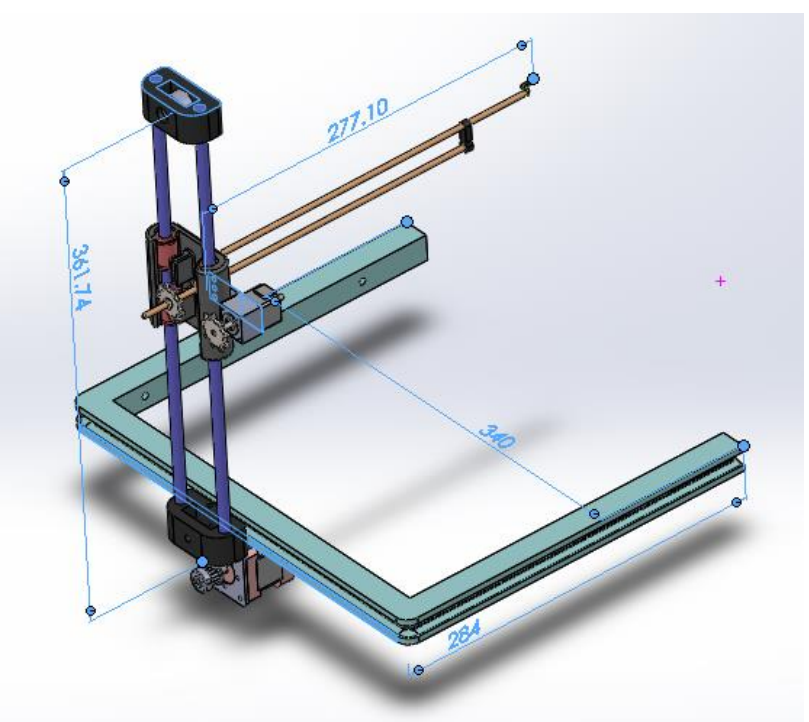

Figure 5: The proposed manipulator to the left (units in $\mathrm{mm}$ ) attached to the $3 \mathrm{D}$-printer body to the right.

It will be actuated as temporal external support while printing to avoid the usage of common support structures and would move around the bed on a chain. The recently added mechanism $(340 \mathrm{~mm}$ $\times 440 \mathrm{~mm} \times 340 \mathrm{~mm}$ ) can move around the $3 \mathrm{D}$ printer bed from the 3 frontal faces with the ability to move vertically in synch with the printing head. Also, it's able to move within the bed area to reach the desired location as revealed in Fig. 6.

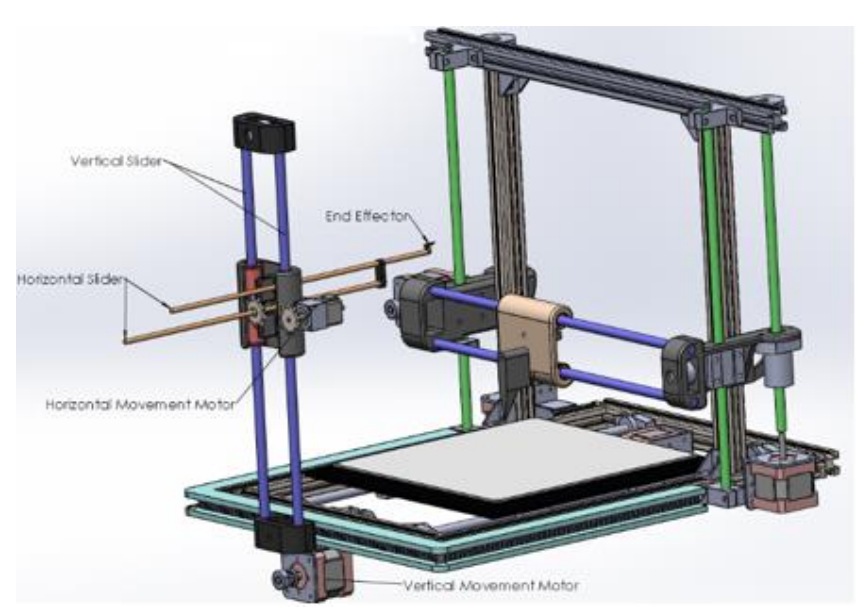

Figure 6: The proposed manipulator to the left (units in $\mathrm{mm}$ ) attached to the $3 \mathrm{D}$-printer body to the right.
A closer look at the end-effector is shown in Fig. 7 with the dimensions (in $\mathrm{mm}$ ). It was designed to be compliant with the motion of the $3 \mathrm{D}$ printer's nozzle. For simplicity, the two actuators responsible for rotating the end-effector in two degrees of freedom are omitted. It is important to highlight that the very bottom layer of the overhanging feature will be printed directly onto the end effector of the robot arm. The freshly extruded material from the FDM nozzle adheres to the end effector, just like how freshly extruded material anchors onto a regular support structure. The end effector of the robot arm is designed with a special slippery surface (made out of glass and supported with heaters to make the filament material adheres on it and coolers to release the material from it). Thus, the freshly extruded material will only be lying on top of the tip of the end-effector rather than adhering into it.

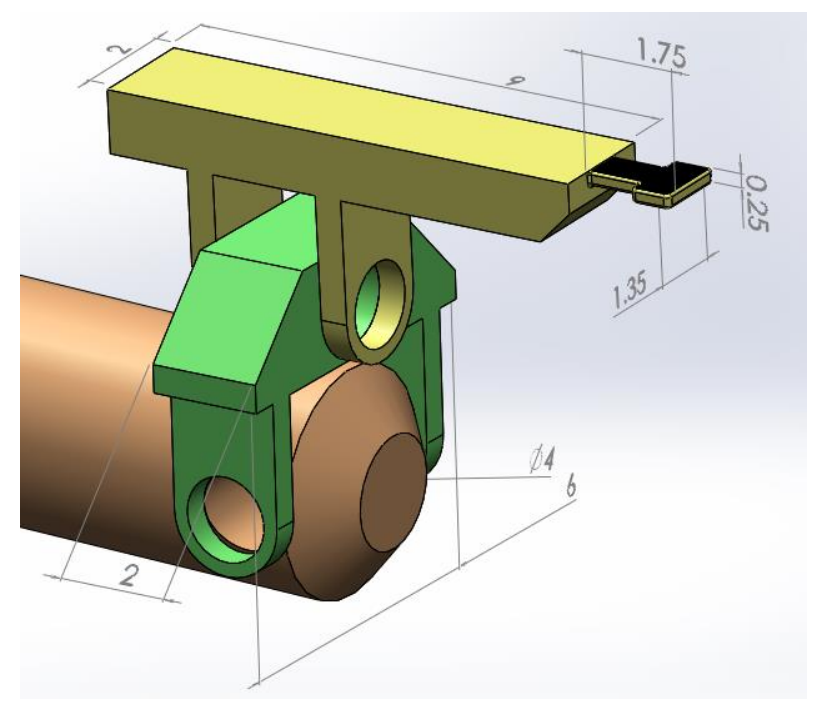

Figure 7: The tip of the added manipulator

\section{Three Dimensional Printing Flowchart}


The program for the upgraded 3D printer will be different from usual printers when support is

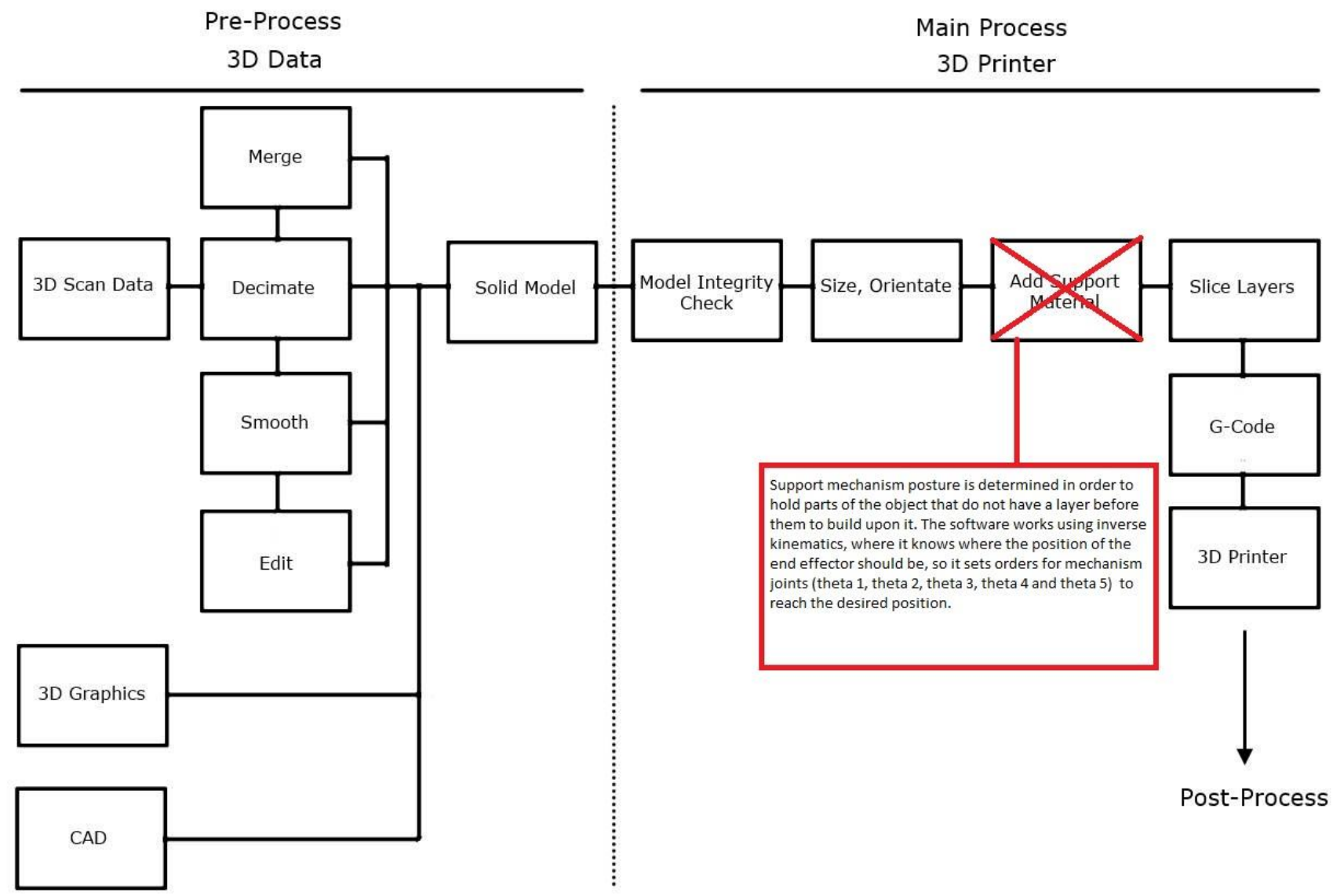

Figure 8: Modified flowchart of the printing process

needed. Instead of adding supports, the software will order the supplementary mechanism to move its end-effector to the location of model-support contact to compensate for the absence of preprinted support structure. The modified flow chart [23] is shown in Fig. 8. Note that the 'Add Support Material' step is replaced with a special step needed to position the end-effector plate under the printing head when support is needed.

Fig.9 illustrates the printing process with the help of the recently proposed mechanism. The robotic arm follows the nozzle of the printer during printing activities to support the printed part of the model that hangs over space.

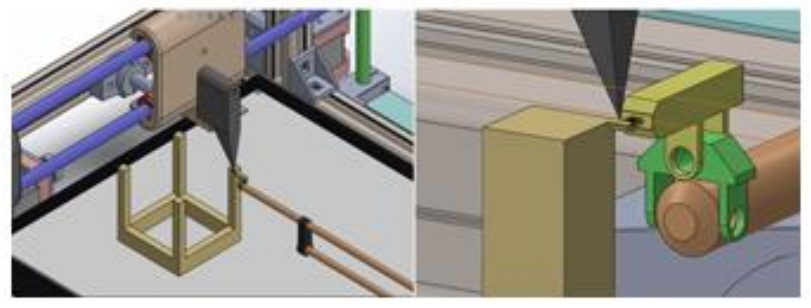

Figure 9: Printing with the help of the robotic arm

\section{Kinematic / Kinetic Description of the Robotic Arm}

The forward kinematic is provided to enhance our understanding of the motion of the new employed mechanism. The derived equations will not only describe the geometry of the system but also allow motion control of the mechanism adequately and smoothly. The mechanism is modelled utilizing links and joints as shown in Fig. 10.

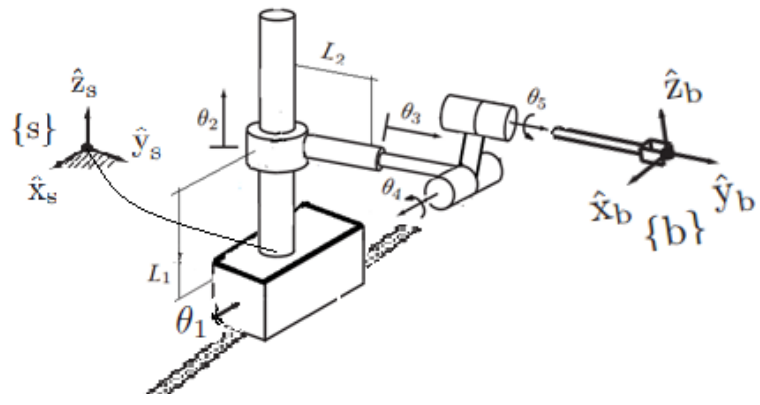

Figure 10: Links and Joints of the added robotic arm. 
The forward kinematics of this robotic mechanism can be expressed using Chasles-Mozzi theorem as a product of exponentials as given in (1):

$$
T(\theta)=e^{\left[S_{1}\right] \theta_{1} \ldots e^{\left[S_{5}\right] \theta_{5} M}}
$$

\section{Where:}

$$
S_{i}=\left(w_{i}, v_{i}\right) \in \mathfrak{R}^{6} \text {, is a six-dimensional screw }
$$
axis along the $i^{\text {th }}$ joint expressed in the fixed frame $\{S\} . w_{i}$ is a unit vector in the direction of the ith screw axis expressed in frame $\{S\}$ when the joint is rotary and zero vector when it's prismatic. ${ }^{v_{i}}$ is the unit vector in the direction of the prismatic joint and when a rotary joint exists then $v_{i}=-w_{i} \times q_{i}+h_{i} w_{i}$. Here, $h$ stands for the screw pitch as a linear or angular speed. $\mathrm{q}$ is an arbitrary point chosen on the screw axis.

Then $S_{i}$ can be represented as $4 \times 4$ matrix denoted as:

$$
\left[S_{i}\right] \equiv\left[\begin{array}{cccc}
0 & -w_{i}^{3} & w_{i}^{2} & v_{i}^{1} \\
w_{i}^{3} & 0 & -w_{i}^{1} & v_{i}^{2} \\
-w_{i}^{2} & w_{i}^{1} & 0 & v_{i}^{3} \\
0 & 0 & 0 & 0
\end{array}\right]
$$

$\theta_{i}$ is the $i^{\text {th }}$ joint variable that can be rotary or prismatic.

$M$ is the end-effector transformation given in $\{S\}$ when the robot is in home configuration.

The use of the above formulation has several advantages over the commonly used approach denoted as Denavit-Hartneberg parameters. One advantage centers on the flexibility of no need to denote link frames associated with a more compact form regardless of the joint type [24].

The above parameters can be defined based on Fig. 11 as follows:

$$
M=\left[\begin{array}{cc}
R_{S b} & p_{S b} \\
0 & 1
\end{array}\right]=\left[\begin{array}{cccc}
1 & 0 & 0 & 0 \\
0 & 1 & 0 & L_{2} \\
0 & 0 & 1 & L_{1} \\
0 & 0 & 0 & 1
\end{array}\right] \in S E(3)
$$

SE(3) stands for Special Euclidean Group of order three.

$R_{S b}$ is the orientation of frame $\{b\}$ in the $\{S\}$ frame in zero position. ${ }^{p}{ }_{S b}$ is the position of frame $\{b\}$ in the $\{S\}$ frame in zero position. Table1 summarizes the whole $S_{i}=\left(w_{i}, v_{i}\right)$ set.

Table1: Screw axes

\begin{tabular}{|l|l|l|l|}
\hline$i$ & $w_{i}$ & $q_{i}$ & $v_{i}$ \\
\hline 1 & $(0,0,0)$ & - & $(1,0,0)$ \\
\hline 2 & $(0,0,0)$ & - & $(0,0,1)$ \\
\hline 3 & $(0,0,0)$ & - & $(0,1,0)$ \\
\hline
\end{tabular}

\begin{tabular}{|c|c|c|c|}
\hline 4 & $(1,0,0)$ & $\left(0, L_{2}, L_{1}\right)$ & $\left(0, L_{1},-L_{2}\right)$ \\
\hline 5 & $(0,1,0)$ & $\left(0,0, L_{1}\right)$ & $\left(-L_{1}, 0,0\right)$ \\
\hline
\end{tabular}

Now, as long as the tip of the manipulator will hold a certain amount of the dispensed polymer material (i.e. molten filament) from the nozel, the torques and forces must be adapted to compensate for the added weight and other forces that may exist by the print head to guarantee a flat top road surface. Then the spatial force acting on the tip in frame $\{s\}$ is given as $F_{s}=\left[\begin{array}{c}m_{s} \\ f_{s}\end{array}\right] \in \mathfrak{R}^{6}$, where $m$ is the moment and $\mathrm{f}$ is the linear force. Note that in case multiple wrenches act on the end effector then a spatial force is represented by their vector summation. Thus, the exerted torques at each joint are given as in Eqn. 4.

$$
\tau=J^{T}(\theta) F_{s}
$$

Where the ith column of Jacobian matrix, $J(\theta)$, is defined as $\left.J_{i}(\theta)=A d_{e^{\left[S_{1}\right] \theta_{1} \ldots}} e^{\left[S_{i-1}\right] \theta_{i-1}}{ }_{i}\right)$, which is the adjoint mapping representation applied at the ith screw axis after being subjected to a rigid body displacement as at arbitrary joint parameters.

When the Jacobian rank is not maximal, then a kinematic singularity does exist. Merely, the Jacobian of the proposed mechanism is derived as follows:

$$
\begin{aligned}
\dot{T}=\frac{d e^{\left[S_{1}\right] \theta_{1}}}{d t} \ldots & \ldots e^{\left[S_{5}\right] \theta_{5}} M \\
& +e^{\left[S_{1}\right] \theta_{1}}\left(\frac{d e^{\left[S_{2}\right] \theta_{2}}}{d t}\right) \ldots \ldots e^{\left[S_{5}\right] \theta_{5}} M \\
& +\cdots \ldots
\end{aligned}
$$

Then

$$
\begin{aligned}
& \dot{T} T^{-1} \\
& =\left[S_{1}\right] \dot{\theta_{1}}+e^{\left[S_{1}\right] \theta_{1}}\left[S_{2}\right] e^{-\left[S_{1}\right] \theta_{1}} \dot{\theta}_{2} \\
& +e^{\left[S_{1}\right] \theta_{1}} e^{\left[S_{2}\right] \theta_{2}}\left[S_{3}\right] e^{-\left[S_{2}\right] \theta_{2}} e^{-\left[S_{1}\right] \theta_{1}} \dot{\theta}_{3}+\cdots \ldots \\
& =\left[S_{1}\right] \dot{\theta_{1}}+\operatorname{Ad}_{e^{\left[S_{1}\right] \theta_{1}}}\left(S_{2}\right) \dot{\theta_{2}}
\end{aligned}
$$

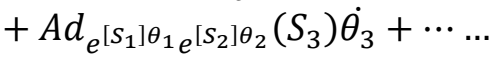

$$
\begin{aligned}
& =\left[\begin{array}{lllr}
J_{S_{1}} & J_{S_{2}}(\theta) & \ldots \ldots & J_{S_{5}}(\theta)
\end{array}\right]\left[\begin{array}{c}
\dot{\theta_{1}} \\
\vdots \\
\dot{\theta}_{5}
\end{array}\right] \\
& J=\left[\begin{array}{ccccc}
0 & 0 & 0 & 1 & 0 \\
0 & 0 & 0 & 0 & \cos \left(\theta_{4}\right) \\
0 & 0 & 0 & 0 & \sin \left(\theta_{4}\right) \\
1 & 0 & 0 & 0 & -L_{1}-\theta_{2} \\
0 & 0 & 1 & L_{1}+\theta_{2} & -\theta_{1} \sin \left(\theta_{4}\right) \\
0 & 1 & 0 & -L_{2}-\theta_{3} & \theta_{1} \cos \left(\theta_{4}\right)
\end{array}\right]
\end{aligned}
$$

Then the spatial twist can be related to the joints variable rate by (6): 


$$
V=J \dot{\theta}
$$

Moreover, by knowing the singularity, appropriate analysis of the Jacobian matrix will help in configuring different situations where the manipulator tip is unable to produce velocities in definite directions. On the other hand, if the tip of the proposed mechanism is required to follow predefined trajectory $[£(t)]$ then it becomes easy to calculate the inverse kinematics $\theta \mathrm{d}(\mathrm{n} \Delta \mathrm{t})$ at each discrete timestamp $\mathrm{n}$ to control the joint velocities as in (7):

$$
\dot{\theta}=\frac{\left[\theta_{d}(n \Delta t)-\theta((n-1) \Delta t)\right]}{\Delta t}
$$

From equation (7), we instilled feedback to the controller by comparing the desired joint angle with the most recently restrained real joint angle.

Alternatively, equation (6) can be rearranged to $(8)$ :

$$
\dot{\theta}=J^{-1} V_{d}
$$

Where the desired twist can be the proposed mechanism twist at the desired trajectory.

During mechanism motion, the controller is provided by a steady stream of target positions and orientations described in (1) and with velocities in (6) and (8) that allow the mechanism track a certain trajectory. This trajectory will be defined by the software that slices the CAD model of the print into layers. An operation signal is then sent to the controller board based on the slices layers. The controller than command the motors of the proposed mechanism, print bed and the extruder.

\section{Conclusion}

This paper is among the efforts in reducing or removing supports in 3D printing operation in correlation to fining degree of textures and granularities that can be achieved. Some suggested upgraded software that can decrease the number of supports, some changed in material characteristics or its type, and we are shedding the light on using the mechanism to decrease the usage of support structures. The usage of CAD/CAM software solution will not remove the supports but will optimize their position. And for the material change, it may become costly more than the current materials 3D printers use. As an advantage, our mechanism works in conjunction with the 3D printer nozzle under the monitoring of updated software to work as in the proposed flowchart to maximize the efficiency of $3 \mathrm{D}$ printing. A mathematical formulation has been introduced along with the mechanism design. The robotic arm has been simulated in SolidWorks along with the 3D printer mechanism.
As a conclusion, such introduced mechanisms can solve the problem of exterior support structures in FDM 3D printers with an acceptable cost. As a matter of fact, we will achieve a better speed in the $3 \mathrm{D}$ printing process and thus saving time and material usage for supports, eliminating the constraints imposed by Earth's gravity. The mechanism is flexible and can adapt to different positions needed for supports. Besides, it can move around the 3D printer bed from three directions and to move within the bed area. It is a wild concept to build a 3D printer with built-in anti-support mechanism, but better results could be achieved by more research and experiments. For instance, the proposed mechanism is not able to replace the overhanging inner wall of an angle pipe structure. Currently, In case of an arc exceeding the ability of the end-effector, supports will be printed as necessary. The software is built to compensate for such scenarios.

As future work, the robot must be equipped with advanced tools to overcome overhanging features presented inside a complex geometry that block the manipulator from reaching the inside.

\section{References:}

[1] D. Espalin, J. Ramierez, F. Medina and R. Wicker, "Multi-Material, Multi-Technology FDM System," University of Texas, El Paso, 2012. [2] J. Vanek, J. A. G. Galicia, B. Benes, "Clever Support: Efficient Support Structure Generation," Eurographics Symposium on Geometry Processing, vol. 33 (2014), no. 5, 2014. [3] W. R. Priedeman, A. L. Brosch and E. Prairie, "Soluble material and process for three-dimensional modeling". United States of America Patent US 6,790,403 B1, 14 September 2004. [4] Greer, C (Greer, Clayton); Nycz, A (Nycz, Andrzej); Noakes, M (Noakes, Mark); Richardson, B (Richardson, Brad); Post, B (Post, Brian); Kurfess, T (Kurfess, Thomas); Love, L (Love, Lonnie), 2019. Introduction to the design rules for Metal Big Area Additive Manufacturing. ADDITIVE MANUFACTURING Volume: 27 Pages: 159-166 DOI: 10.1016/j.addma.2019.02.016. [5] M. Leary, L. Merli, F. Torti, M. Mazur and M. Brandt, "Optimal topology for additive manufacture: A method for enabling additive manufacture of support-free optimal structures," Materials and Design, Elsevier, vol. 63, pp. 678-690, 2014. [6] Zhao, D., \& Guo, W. (2019). Mixed-layer adaptive slicing for robotic Additive Manufacturing (AM) based on decomposing and regrouping. 
Journal of Intelligent Manufacturing. doi:10.1007/s10845-019-01490-z

[7] D. Ong U Jing, D. M. Devine and J. Lyons, "3D Printed End of Arm Tooling (EOAT) for Robotic Automation," Robotics Journal, Mdpi, vol. 7, no. 49, 2018.

[8] O. Kontovourkisa and G. Tryfonos, "Integrating parametric design with robotic additive manufacturing for 3D clay printing: An experimental study," in 35th International Symposium on Automation and Robotics in Construction (ISARC 2018), 2018.

[9] I. Ishak, J. Fisher and P. Larochelle, "Robot Arm Platform for Rapid Prototyping: Concept," in Florida Conference on Recent Advances in Robotics, FCRAR 2015, Melbourne, Florida, 2015.

[10] I. Ishak and P. Larochelle, "Robot Arm Platform for Additive Manufacturing: 3D Lattice Structures," in 30th Florida Conference on Recent Advances in Robotics, Florida Atlantic University, Boca Raton, Florida, May 11-12, 2017.

[11] M. Efe Tiryaki, X. Zhang and Q.-C. Pham, "Printing-while-moving: a new paradigm for largescale robotic 3D Printing," in arXiv:1809.07940v1, 21 Sep 2018.

[12] P. Urhal, A. Weightman, C. Diver, P. Bartolo, Robot assisted additive manufacturing: a review, Rob. Comput. Integr. Manuf. 59 (2019) 335-345..

[13] S. Hongyao, P. Lingnan and Q. Jun, "Research on large-scale additive manufacturing based on multi-robot collaboration technology," Additive Manufacturing, vol. 30, no. https://doi.org/10.1016/j.addma.2019.100906, 2019.

[14] R. Alkhatib, W. Mechlawi and R. Kawtharani, "Quality Assessment of Robotic Grasping Using Regularized Logistic Regression," in IEEE Sensors Letters, vol. 4, no. 6, pp. 1-4, June 2020, Art no. 5500904, doi: 10.1109/LSENS.2020.2994166.

[15] Scalera, L.; Palomba, I.; Wehrle, E.; Gasparetto, A.; Vidoni, R. Natural Motion for Energy Saving in Robotic and Mechatronic Systems. Appl. Sci. 2019, 9, 3516.

[16] Carabin, G., Scalera, L., Wongratanaphisan, T., \& Vidoni, R. (2021). An energy-efficient approach for 3D printing with a Linear Delta Robot equipped with optimal springs. Robotics and ComputerIntegrated Manufacturing, 67, 102045. doi:10.1016/j.rcim.2020..

[17] Espera, A.H., Dizon, J.R.C., Chen, Q. et al. 3Dprinting and advanced manufacturing for electronics. Prog Addit Manuf 4, 245-267 (2019). https://doi.org/10.1007/s40964-019-00077-7.
[18] Jahan Zeb Gul, Memoon Sajid, Muhammad Muqeet Rehman, Ghayas Uddin Siddiqui, Imran Shah, Kyung-Hwan Kim, Jae-Wook Lee \& Kyung Hyun Choi (2018) 3D printing for soft robotics - a review, Science and Technology of Advanced Materials, 19:1, 243-262, DOI: 10.10.

[19] Shembekar, A. V., Yoon, Y. J., Kanyuck, A., and Gupta, S. K. (April 1, 2019). "Generating Robot Trajectories for Conformal Three-Dimensional Printing Using Nonplanar Layers." ASME. J. Comput. Inf. Sci. Eng. September 2019; 19(3): 031011, no. https://doi.org/10.1115/1.4043013.

[20] Subrin, K., Bressac, T., Garnier, S., Ambiehl, A., Paquet, E., \& Furet, B. (2018). Improvement of the mobile robot location dedicated for habitable house construction by $3 \mathrm{D}$ printing. IFACPapersOnLine, 51(11), 716-721. doi:10.1016/j.ifacol.2018.08.403.

[21] Joyee, E. B., \& Pan, Y. (2020). Additive manufacturing of multi-material soft robot for ondemand drug delivery applications. Journal of Manufacturing Processes, 56, 1178-1184. doi:10.1016/j.jmapro.2020.03.059 .

[22] Freek Bos, Rob Wolfs, Zeeshan Ahmed \& Theo Salet (2016) Additive manufacturing of concrete in construction: potentials and challenges of 3D concrete printing, Virtual and Physical Prototyping, 11:3, 209-225, DOI: 10.1080/17452759.2016.1209867.

[23] B. J. Rao, "3D Printing a More Tangible Idea," 7 January 2014. [Online]. Available: http://heywhatsthebigidea.net/about-me/articles/3dprinting-tangible-idea/.

[24] Al Hajjar, A., \& Alkhatib, R. (2017). Agile Locomotion. International Conference on Bioengineering for Smart Technologies (BioSMART 2016). Dubai, United Arab Emirates: IEEE.

\section{Author Contributions:}

Nassim Khalid carried out the simulation and testing of the proposed mechanism

Rami Alkharib carried the derivation of the mathematical equations.

Anas Al-Shaghouri carried out the experimentations and the verification of mathematical equations.

Saja Khatib was responsible for the manipulator modelling.

\section{Creative Commons Attribution License 4.0 (Attribution 4.0 International, CC BY 4.0)}

This article is published under the terms of the Creative Commons Attribution License 4.0

https://creativecommons.org/licenses/by/4.0/deed.en_US 